\title{
GENERALIZATIONS OF RESULTS OF AGMON AND NIRENBERG TO THE CAUCHY PROBLEM FOR EVOLUTION EQUATIONS
}

\author{
MONTY J. STRAUSS ${ }^{1}$
}

\begin{abstract}
Uniqueness and norm convexity for evolution equations are investigated by using tools developed by Agmon and Nirenberg.
\end{abstract}

We continue here our study of uniqueness for the Cauchy problem for evolution equations begun in [7]. We investigate

$$
\begin{aligned}
L u(x, t) & =D_{t} u(x, t)+i\left(B\left(x, t, D_{x}\right)+i A\left(x, t, D_{x}\right)\right) u(x, t) \\
& =-i\left[u_{t}(x, t)-\left(B\left(x, t, D_{x}\right)+i A\left(x, t, D_{x}\right)\right) u(x, t)\right],
\end{aligned}
$$

where $B$ and $A$ are pseudo-differential operators in the $x$-variables for $(x, t) \in R^{n} \times R^{1}$ whose principal symbols are real, $D_{x_{j}}=-i \partial / \partial x_{j}$, and $D_{t}=-i \partial / \partial t$. In [7], we examined the equation $L u=0$ for those $B$ that were convolution operators in the $x$ variables, i.e. $B$ independent of $t$. Here we will allow $B$ to depend on $x$, but restrict it in another fashion. In addition to proving uniqueness and a (sharper) norm convexity result as in the previous investigation, we are able to deduce a formula on decay of solutions.

Our work is based on some results of Agmon and Nirenberg ([1], [2], [3]), which in turn are related to the Hadamard Three-Circle Theorem, and we will only indicate how the proofs follow from their work. We use pseudo-differential operators; for their properties see [5].

Our results are stated for single equations. They are also valid for diagonal systems whose component equations satisfy the hypotheses. For details see [6].

Received by the editors December 2, 1971.

AMS 1970 subject classifications. Primary 35A05, 35B30, 58G15; Secondary 35B05.

Key words and phrases. Uniqueness, Cauchy problem, evolution equation, pseudodifferential operator.

1 This material is an extension of some of the results of the author's Ph.D. thesis, written under the direction of Professor Louis Nirenberg at the Courant Institute of New York University on a National Science Foundation Graduate Fellowship. 
THEOREM 1. Suppose that $u(x, t) \in C^{\infty}\left(\Omega \times\left[t_{0}, t_{1}\right)\right)$ with compact support in $\Omega$ for each fixed $t$, where $\Omega \subset R^{n}$, and satisfies

$$
u_{t}(x, t)-\left(B\left(x, t, D_{x}\right)+i A\left(x, t, D_{x}\right)\right) u(x, t)=f, \quad\|f\| \leqq \Phi(t)\|u\|,
$$

where $B$ and $A$ are first order pseudo-differential operators whose principal symbols are real, $\Phi$ is nonnegative, bounded, continuous, and integrable in $t$ and $\|\quad\|=\|\|_{L^{2}\left(R^{n}\right)}$. Assume also that one of the following is valid for $t \in\left[t_{0}, t_{1}\right)$ :

(a) $B$ is elliptic of order one; i.e. $\|\Lambda v\| \leqq C(t)(\|B v\|+\|v\|)$ for all $v$ in $C_{0}^{\infty}(\Omega)$, where $\Lambda=(1-\Delta)^{1 / 2}, \Delta=$ Laplacian;

(b) $B$ is of order zero instead of order one;

(c) $\left\|B_{t} v\right\| \leqq C(t)\|B v\|$ for all $v$ in $C_{0}(\Omega)$, where $B_{t}=d B / d t$; or

(d) the principal symbol $g$ of $B_{t}$ is nonnegative.

Then if $\|u(\bar{t})\|=0$ for $i \in\left[t_{0}, t_{1}\right)$, $u$ vanishes identically in $\Omega \times\left[t_{0}, t_{1}\right)$. Also, if $\|u(t)\| \neq 0$ for all $t \in\left[t_{0}, t_{1}\right)$, then $\|u(t)\| \geqq\left\|u\left(t_{0}\right)\right\| \exp \left[-\mu\left(t-t_{0}\right)\right]$ for $t_{1}>t \geqq t_{0}+1$, where $\mu$ is a constant independent of $t$. Finally, if $u(t) \neq 0$ for all $t \in\left[\tau_{0}, \tau_{1}\right)$, where $t_{0} \leqq \tau_{0} \leqq t<\tau_{1} \leqq t_{1}$, then

$$
\log \|u(t)\| \leqq \log \left\|u\left(\tau_{0}\right)\right\| \frac{k\left(\tau_{1}\right)-k(t)}{k\left(\tau_{1}\right)-k\left(\tau_{0}\right)}+\log \left\|u\left(\tau_{1}\right)\right\| \frac{k(t)-k\left(\tau_{0}\right)}{k\left(\tau_{1}\right)-k\left(\tau_{0}\right)}+N,
$$

where $k(\tau)=\int_{\tau_{0}}^{\tau} \exp \left(\mp \int_{\tau_{0}}^{s} M(\lambda) d \lambda\right) d s, M(\lambda)$ is a nonnegative, continuous, and integrable function on $\left[\tau_{0}, \tau_{1}\right)$ that is independent of $u$, the + sign is used if $\left\|u\left(\tau_{0}\right)\right\| \geqq\left\|u\left(\tau_{1}\right)\right\|$ and the - sign if $\left\|u\left(\tau_{0}\right)\right\| \leqq\left\|u\left(\tau_{1}\right)\right\|$, and $N$ is a nonnegative constant independent of $u$.

Proof. This result follows from Agmon and Nirenberg's work [1, Théorème 2.1, Théorème 2.2, Proposition 2.1, Proposition 2.2] after verification of the three principal hypotheses of Agmon and Nirenberg in each of (a)-(d).

A-N 0. $B=B_{+}+B_{-}$, where $\left(B_{+} v, w\right)=\left(v, B_{+} w\right)$ and $\operatorname{Re}\left(B_{-} v, v\right)=0$ for all $v, w$ in $C_{0}(\Omega)$ and each $t$ in $\left[t_{0}, t_{1}\right)$, and each of $B_{+}$and $B_{-}$are smooth;

A-N I. $\quad \operatorname{Re}\left(B_{+} u, B_{-} u\right) \geqq-C(t)\left(\left\|B_{+} u\right\|+\|u\|\right) ;$ and

A-N II. $\quad(d / d t)\left(B_{+} u, u\right)-2 \operatorname{Re}\left(B_{+} u, u_{t}\right) \geqq C(t)\|u\|\left(\left\|B_{+} u\right\|+\|u\|\right)$, or alternately, $\left\|\left(B_{+}\right)_{t} u\right\| \leqq C\left(\left\|B_{+} u\right\|+\|u\|\right)$.

We may assume again that $A \equiv 0$ by a technique due to Egorov (see [6], [7]). Then set $B_{+}=\frac{1}{2}\left(B+B^{*}\right)$ and $B_{-}=\frac{1}{2}\left(B-B^{*}\right)$, where $\left(B^{*} v, w\right) \equiv$ $\left(v, B w^{\prime}\right)$ for all smooth $v$ and $w$ and the hypotheses of Agmon-Nirenberg are easily seen to be satisfied. Q.E.D.

REMARK 1. Hypothesis (c) includes the case where the principal symbol $b$ of $B$ is independent of $t$. 
REMARK 2. The convexity inequality is equivalent to the following formula:

$$
\|u(t)\| \leqq e^{N}\left\|u\left(\tau_{0}\right)\right\|^{1-\alpha(t)}\left\|u\left(\tau_{1}\right)\right\|^{\alpha(t)},
$$

where $0 \leqq \alpha(t) \nearrow 1$ for $\tau_{0} \leqq t \nearrow \tau_{1}$, and $\alpha(t)$ is an integral expression. If $N=0$ and $\alpha$ is linear in $t$, then this would be a standard convexity result:

or

$$
\|u(t)\| \leqq\left\|u\left(\tau_{0}\right)\right\|^{\left(\tau_{1}-t\right) /\left(\tau_{1}-\tau_{0}\right)}\left\|u\left(\tau_{1}\right)\right\|^{\left(t-\tau_{0}\right) /\left(\tau_{1}-\tau_{0}\right)},
$$

$$
\begin{aligned}
\log \|u(t)\| \leqq & \left(\left(\tau_{1}-t\right) /\left(\tau_{1}-\tau_{0}\right)\right) \log \left\|u\left(\tau_{0}\right)\right\| \\
& +\left(\left(t-\tau_{0}\right) /\left(\tau_{1}-\tau_{0}\right)\right) \log \left\|u\left(\tau_{1}\right)\right\| .
\end{aligned}
$$

We may relax the restrictions on $B$ in Theorem 1 slightly.

Proposition 2. Suppose that, instead of satisfying one of (a)-(d), B fulfills

(e) $B\left(x, t, D_{x}\right)=h(t) B_{0}\left(x, D_{x}\right)$, where $h(t)$ is a continuous nonnegative function of $t$ alone with zeros of finite order in $\left[t_{0}, t_{1}\right)$ such that $\lim _{t \wedge t_{1}} h(t) \neq$ $0, B_{0}\left(x, D_{x}\right)$ is an arbitrary scalar Hermitian first order pseudo-differential operator and, in addition to $\|f\| \leqq \Phi(t)\|u\|$, the function $f$ satisfies $f=$ $O(h(t))$ for $h(t) \searrow 0$ and $f / h$ is integrable with respect to $t$ for each fixed $x$.

Then the results of Theorem 1 remain valid.

Proof. We suppose without loss of generality that $h\left(t_{0}\right)=0$, as we can always subdivide the $t$-interval and prove the result for each subinterval if $h(t)=0$ for some $t \in\left(t_{0}, t_{1}\right)$ and $h\left(t_{0}\right) \neq 0$. [If $h(t)$ is bounded away from 0 in $\left[t_{0}, t_{1}\right)$, then there is nothing to show.] We have

$$
u_{t}(x, t)-h(t) B_{0}\left(x, D_{x}\right) u(x, t)=f(x, t) .
$$

Let $s(t)=\int_{\tau_{0}}^{t} h(\lambda) d \lambda$. Then $s$ is a differentiable monotone increasing function on $\left[t_{0}, t_{1}\right)$ and thus has a continuous monotone increasing inverse $t=\phi(s)$. We also define $v(x, s)=u(x, t)$. Then $v(x, s)$ is $C^{\infty}$ in $\Omega \times\left[0, s\left(\tau_{1}\right)\right)$ and $v$ vanishes outside $\Omega$ for each fixed $s$. Since $u(x, t)=$ $v\left(x, \int_{\tau_{0}}^{t} h(\lambda) d \lambda\right)$, we get

by the chain rule.

$$
u_{t}(x, t)=v_{s}\left(x, \int_{\tau_{0}}^{t} h(\hat{\lambda}) d \lambda\right) h(t)
$$

Then (2) is equivalent to

$$
v_{s}(x, s)-B_{0}\left(x, D_{x}\right) v(x, s)=f(x, t) / h(t) \equiv f_{1}(x, s) .
$$

Now, $f(x, t)=O(h(t))$ for $h(t) \downarrow 0$ implies that $\left\|f_{1}(x, s)\right\| \leqq \Phi_{1}(s)\|u(s)\|$, where $\Phi_{1}$ satisfies the same properties as $\Phi$, and $B_{0}\left(x, D_{x}\right)$ satisfies (c) in Theorem 1: the principal symbol $g$ of $B_{0}$ is nonnegative. Therefore the results of the theorem hold with $s$ replaced by $t$, and, since $s$ is merely a rescaling of the $t^{2}$ axis, we obtain the conclusions we desire. Q.E.D. 
Two types of "pseudo-parabolic" equations can also be handled by the technique of Agmon-Nirenberg.

THEOREM 3. Suppose that instead of one of (a)-(d) holding, B is a Hermitian scalar-valued elliptic second order pseudo-differential operator. Then the conclusions of Theorem 1 are again valid.

Proof. This time we satisfy an alternative set of conditions. A-N 0, A-N I', and A-N II, where

AN I'. $\left\|B_{-} u\right\|^{2} \leqq C(t)\|u\|\left(\left\|B_{+} u\right\|+\|u\|\right)$ replaces A-N I. We define $B_{+}$and $B_{-}$as before: $B_{+}=\frac{1}{2}\left(B+B^{*}\right)=B+H$, where $H$ is a first order pseudo-differential operator, and $B_{-}=\frac{1}{2}\left(B-B^{*}\right)=-H$. Then $\mathrm{A}-\mathrm{N} 0$ is satisfied by definition. Since $B$ is elliptic of order two, we have

$$
\begin{array}{rlr}
\left\|\Lambda^{2} u\right\| & \leqq C_{0}\|B u\|+C\|\Lambda u\|, \quad \text { where } \Lambda=(1-\Delta)^{1 / 2}, \\
& \leqq C_{0}\left\|B_{+} u\right\|+C\|H u\|+C\|\Lambda u\| \\
& \leqq C_{0}\left\|B_{+} u\right\|+C_{1}\|\Lambda u\| .
\end{array}
$$

Given $\varepsilon>0$, we can choose $C_{\varepsilon}=1 / \varepsilon$ such that $\|\Lambda u\| \leqq \varepsilon\left\|\Lambda^{2} u\right\|+C_{\varepsilon}\|u\|$. Choose $\varepsilon$ so that $C_{1} \varepsilon<\frac{1}{2}$ for $t$ in $\left[t_{0}, t_{1}\right)$. Then

$$
\left\|\Lambda^{2} u\right\| \leqq C_{0}\left\|B_{+} u\right\|+C_{1} \varepsilon\left\|\Lambda^{2} u\right\|+C_{\varepsilon}\|u\|+C_{2}\|u\| ;
$$

i.e., $\left\|\Lambda^{2} u\right\| \leqq 2 C_{0}\left\|B_{+} u\right\|+2\left(C_{\varepsilon}+C_{2}\right)\|u\|$. Thus

$$
\begin{aligned}
\left\|B_{-} u\right\|^{2} & =(H u, H u) \leqq C(\Lambda u, \Lambda u)=C\left|\left(\Lambda^{2} u, u\right)\right| \\
& \leqq\left\|\Lambda^{2} u\right\|\|u\| \leqq C\|u\|\left(\left\|B_{+} u\right\|+\|u\|\right),
\end{aligned}
$$

which is $\mathrm{A}-\mathrm{N} \mathrm{I}^{\prime}$.

For A-N II,

$$
\left\|\left(B_{+}\right)_{t} u\right\| \leqq C\left\|\Lambda^{2} u\right\| \leqq C\left\|B_{+} u\right\|+C\|u\| .
$$

Therefore the hypotheses of Agmon-Nirenberg are satisfied and the theorem holds. Q.E.D.

The next result differs from Theorem 3 in that we allow second order differentiation in $t$, but use only first order pseudo-differential operators.

THEOREM 4. Suppose that

$$
\left\|u_{t}-B_{1} u\right\| \leqq \Phi(t)\left\{\left\|u_{t}\right\|^{2}+\operatorname{Re}\left(B_{1} u, u\right)+M_{B}(t)(u, u)\right\}^{1 / 2},
$$

for $u \in C^{\infty}\left(\Omega \times\left[t_{1}, t_{2}\right)\right)$ with compact support in $\Omega$ for each fixed $t$, where $B_{1}$ is a real first order positive semidefinite pseudo-differential operator, $\Phi(t)$ is as before, and the function $M_{B}(t)$, which is continuous and integrable in $t$ and satisfies $M_{B}(t)>1$, comes from Gårding's inequality [4]: $\operatorname{Re}\left(B_{1} u, u\right) \geqq-\left(M_{1}-1\right)\|u\|^{2}$. Set $B=\frac{1}{2}\left(B_{1}+B_{1}^{*}\right)+M_{B}$ and assume moreover that $\left|\left(B_{t} u, u\right)\right| \leqq C|(B u, u)|$ and $\left\|B_{t} u\right\| \leqq C\|B u\|$. 
If $\left\|u_{t}\right\|^{2}+(B u, u)=0$ for some $i \in\left[t_{1}, t_{2}\right)$, then $u_{t} \equiv 0$ and $(B u, u) \equiv 0$ for all $t$ in the interval. This implies that $u(t)=$ constant, and since $B$ is positive definite, then $u \equiv 0$ in $\Omega \times\left[t_{1}, t_{2}\right)$.

Proof. This is a direct result of Théorème 3.1 and Corollaire 3.1 in [1].

\section{BIBLIOGRAPHY}

1. S. Agmon, Unicité et convexité dans les problèmes différentiels, Séminaire de Mathématiques Supérieures, no. 13 (Été, 1965), Les Presses de l'Université de Montréal, Montréal, Que., 1966. MR 40 \#6025.

2. S. Agmon and L. Nirenberg, Properties of solutions of ordinary differential equations in Banach space, Comm. Pure Appl. Math. 16 (1963), 121-239. MR 27 \#5142.

3. - Lower bounds and uniqueness theorems for solutions of differential equations in Hilbert space, Comm. Pure Appl. Math. 20 (1967), 207-229. MR 34 \#4665.

4. P. D. Lax and L. Nirenberg, On stability for difference schemes: $A$ sharp form of Gårding's inequality, Comm. Pure Appl. Math. 19 (1966), 473-492. MR 34 \#6352.

5. L. Nirenberg, Pseudo-differential operators, Proc. Sympos. Pure Math., vol. 16, Amer. Math. Soc., Providence, R.I., 1970, pp. 149-167. MR 42 \#5108.

6. M. Strauss, Uniqueness and norm convexity for the Cauchy problem, Thesis, New York University, 1971.

7. - Uniqueness and norm convexity in the Cauchy problem for evolution equations with convolution operators, Proc. Amer. Math. Soc. 35 (1972), 423-430.

Department of Mathematics, Texas Tech University, Lubbock, TeXas 79409 